\title{
Equações para Estimativa do Diâmetro de Copa para Acácia-negra
}

\author{
Carlos Roberto Sanquetta ${ }^{1}$, Alexandre Behling ${ }^{1}$, Ana Paula Dalla Corte ${ }^{1}$, \\ Anabela Couto Fernandes ${ }^{1}$, Sara Moreira Beckert ${ }^{1}$, Augusto Arlindo Simon ${ }^{2}$ \\ ${ }^{1}$ Departamento de Ciências Florestais, Universidade Federal do Paraná - UFPR, Curitiba/PR, Brasil \\ ${ }^{2}$ Departamento Florestal, TANAC S.A., Montenegro/RS, Brasil
}

\section{RESUMO}

A fonte de energia de uma árvore provém da radiação solar e, dessa forma, a copa pode ser considerada o órgão da árvore que influencia na interceptação da energia fotossintética e, consequentemente, no crescimento da árvore. A influência do local de plantio, da idade do povoamento e dos elementos meteorológicos na relação entre o diâmetro de copa (DC) e o diâmetro a 1,30 metro do solo (DAP) da espécie Acacia mearsnii De Wild. foi alvo do presente estudo. Para tanto, um estudo da relação do DC com o DAP foi conduzido em povoamentos de acácia-negra, variando nas idades de 1 a 7 anos e em dois locais de cultivo. O DC é maior na medida em que aumenta o DAP, de forma a atingir uma relação logarítmica. O incremento em DC em função do DAP tendeu a diminuir em diferentes intensidades, sendo para as árvores com menores DAP e vice e versa. A relação de DC e DAP, ao longo da idade do povoamento e entre os locais de cultivos estudados, não foi a mesma, produzindo efeitos dependentes para esses fatores. As equações genéricas geradas para descrever tal relação podem ser utilizadas para a estimativa do DC, sendo os coeficientes correlacionados com a precipitação e a temperatura média máxima do ar.

Palavras-chave: variável Dummy, idade do povoamento, variáveis ambientais.

\section{Equations for Estimate of the Crown Diameter for the Black Wattle}

\begin{abstract}
The energy source of a tree comes from solar radiation, and the crown is considered the tree organ that influences the interception of photosynthetic energy and, consequently, its growth. The influence of growth location, age of population, and meteorological elements on the relationship between crown diameter (CD) and diameter at breast height (DBH) of Acacia mearnsii De Wild. (black wattle) was studied. To this end, the study of the relationship between $\mathrm{CD}$ and $\mathrm{DBH}$ was conducted in stands of 1-7-year-old black wattle trees in two cultivation sites. $\mathrm{CD}$ increases proportionally to the increase in $\mathrm{DBH}$, following a logarithmic relationship. The increase in $\mathrm{CD}$ as a function of $\mathrm{DBH}$ tended to decrease at different intensities, with the largest relative $\mathrm{CD}$ increase for the smallest $\mathrm{DBH}$ increase, and vice versa. The relationship between $\mathrm{CD}$ and $\mathrm{DBH}$ for the different ages and locations of the stands varied, generating dependent effects for these factors. The general equations generated to describe this relationship can be used for CD estimate, and their coefficients are correlated with rainfall and average maximum air temperature.
\end{abstract}

Keywords: dummy variable, age of population, environmental variables. 


\section{INTRODUÇÃO}

A fonte de energia de uma árvore provém da radiação solar, que é transformada pelo processo da fotossíntese em energia química; dessa forma, podese considerar que a copa é o órgão da árvore que influencia na interceptação da energia fotossintética e, consequentemente, no crescimento da árvore.

Segundo Leite et al. (2012), as dimensões da copa interferem na realização dos processos fisiológicos e, muitas vezes, estas são usadas como indicadoras da capacidade de uma árvore de competir por recursos. Características, como o comprimento, o diâmetro e o volume da copa, servem como indicadores de respiração fotossintética e da capacidade de transpiração (Sprinz \& Burkhart, 1987 apud Leite et al., 2012), e como componentes de índices de concorrência (Tomé \& Burkhart, 1989; Biging \& Dobbertin, 1992 apud Leite et al., 2012). Portanto, pode-se inferir que essas variáveis estão diretamente relacionadas com o crescimento e a produção de biomassa (Nutto et al., 2001).

A quantidade de radiação solar disponível no nível do solo, no interior de povoamentos de acácianegra (Acacia mearsnii De Wild.), é uma variável importante em estudos, pois são comuns cultivos em consórcios com a referida espécie. Essa variável é uma função de uma série de fatores, tais como: características morfométricas e fisiológicas, hábitos de crescimento, ângulos foliares e disposição das folhas da espécie (Monteith \& Unsworth, 1990). Além disso, é também determinada pelas características da copa, tais como o diâmetro, e também, conforme destacam Miller \& Normam (1971), pela localização e pelo tamanho dos espaços existentes no dossel, comumente denominados sunflecks.

O diâmetro da copa pode ser considerado como variável básica na estimativa de outras variáveis das árvores, correspondendo à distância entre as linhas de projeção dos pontos mais externos da copa. Em trabalhos, como os de Spiecker (1983), Röhle (1986) apud Nutto et al. (2001), são descritos os problemas relativos a esse tipo de levantamento de dados, concluindo que se deve buscar um equilíbrio entre os gastos de tempo e os recursos financeiros, e a precisão da medição.
As relações morfométricas de uma espécie florestal fornecem os subsídios básicos para as estimativas da biomassa de copa. Variáveis, como a altura total da árvore, a altura de copa e o diâmetro de copa, podem ser facilmente correlacionadas com o diâmetro a 1,30 metro do solo (DAP) (Orellana \& Koehler, 2008; Sanquetta et al., 2011; Costa, 2011).

$\mathrm{O}$ presente trabalho teve como objetivo o desenvolvimento de relações morfométricas para predição dos diâmetros de copa para a espécie Acacia mearnsii De Wild., em espécimes plantados em diferentes sítios e com variáveis independentes de simples obtenção. Adicionalmente, também, propôsse a avaliar a influência de variáveis meteorológicas nessas relações morfométricas.

\section{METODOLOGIA}

Para o desenvolvimento do trabalho, foram utilizados dados de plantios comerciais de acácianegra situados em duas regiões do Estado do Rio Grande do Sul, que concentram a maior área de plantio da espécie: Encosta do Sudeste e Serra do Sudeste, respectivamente, nos municípios de Cristal e Piratini (Fortes, 1956).

No município de Cristal, os povoamentos estão sob as coordenadas centrais Sul $30^{\circ} 55^{\prime}$ S e $52^{\circ} 10^{\prime} \mathrm{W}$. No município de Piratini, os povoamentos estão sob as coordenadas centrais Sul $31^{\circ} 24^{\prime} \mathrm{S}$ e Oeste $52^{\circ} 57^{\prime}$ W (Figura 1).

Os solos do município de Cristal foram classificados como Neossolo Regolítico distrófico e os de Piratini, como Neossolo Litólico distrófico, conforme Mochiutti (2007). As características químicas do horizonte superficial dos solos $(0-10 \mathrm{~cm})$ dos povoamentos estão apresentadas na Tabela 1.

Nos dois locais, os plantios foram estabelecidos tanto em áreas novas (primeira rotação do povoamento) como em áreas de reformas (segunda rotação do povoamento). Para todos os casos, o preparo do solo foi realizado na linha de plantio (cultivo mínimo), com uma subsolagem com três hastes a 40 centímetros de profundidade, e duas gradagens. Os plantios de um ano foram implantados com o espaçamento de $3,0 \mathrm{~m} \times 1,75 \mathrm{~m}$ (1.904 plantas por hectare) e 3,0 $\mathrm{m} \times 1,5 \mathrm{~m}$ para as demais idades 


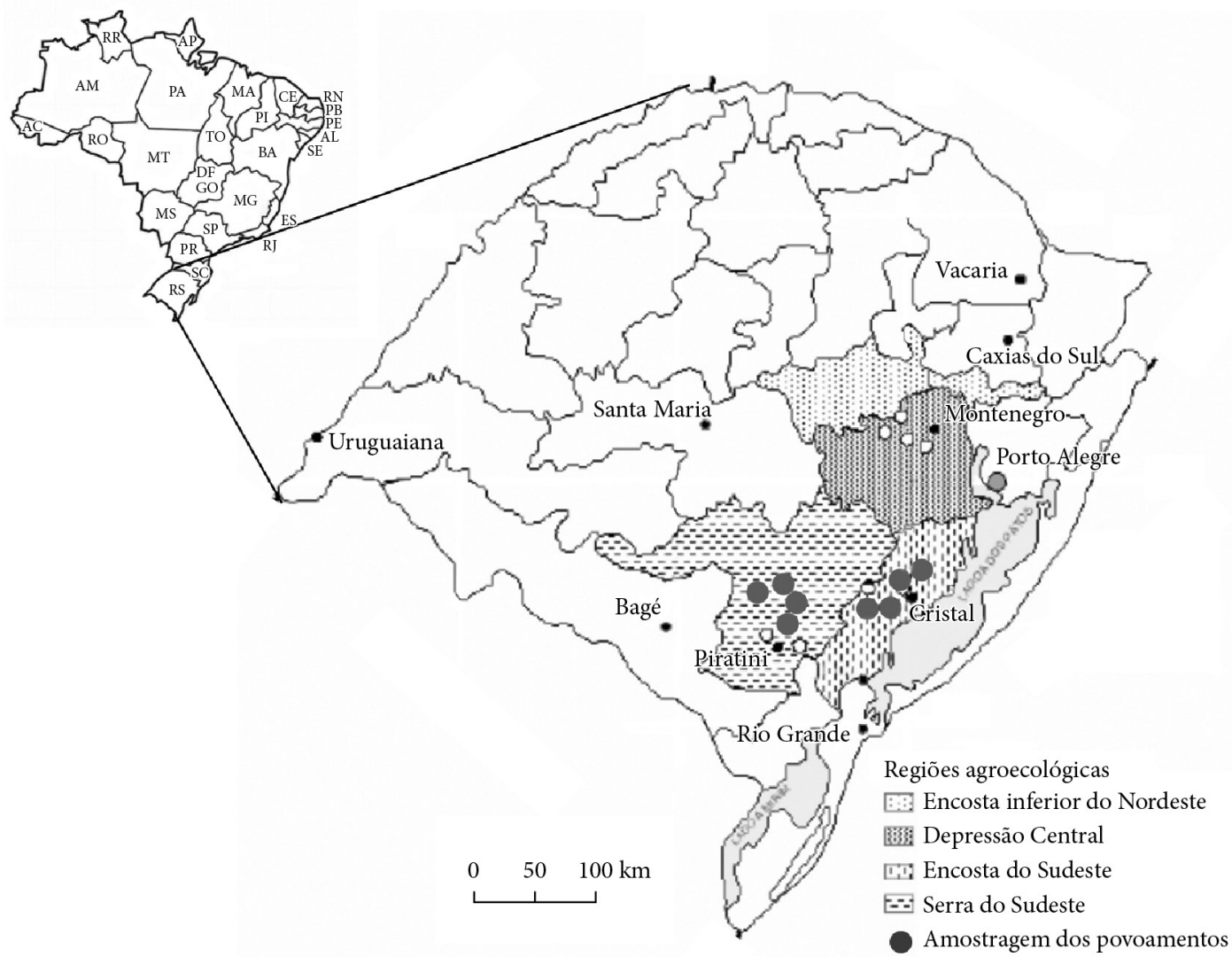

Figura 1. Localização das áreas de estudo no Rio Grande do Sul. Fonte: Adaptado de Rio Grande do Sul (1994).

Figure 1. Location of study áreas on Rio Grande do Sul. Source: Adapted Rio Grande do Sul (1994)

Tabela 1. Características químicas do horizonte superficial do solo em povoamentos de acácia-negra situados em dois municípios do Estado do Rio Grande do Sul.

Table 1. Chemical characteristics of the surface horizon in black wattle stands of varying age located in two municipalities in the State of Rio Grande do Sul.

\begin{tabular}{|c|c|c|c|c|c|c|c|c|c|c|c|c|c|c|}
\hline \multirow{2}{*}{ Região } & \multirow{2}{*}{ Idade } & \multirow{2}{*}{ pH } & \multirow{2}{*}{$\begin{array}{l}\text { IND. } \\
\text { SMP }\end{array}$} & Argila & M. 0. & $\mathbf{P}$ & $\mathbf{K}$ & Al & $\mathrm{Ca}$ & Mg & $\mathbf{H}+\mathbf{A l}$ & $\begin{array}{l}\text { CTC } \\
\text { pH7 }\end{array}$ & \multicolumn{2}{|c|}{ \% Sat. da CTC } \\
\hline & & & & \multicolumn{2}{|c|}{$\%$} & \multicolumn{2}{|c|}{$\mathrm{mg} / \mathrm{L}$} & \multicolumn{5}{|c|}{ cmolc/L } & Bases & Al \\
\hline Cristal & 1 & 4,90 & 5,80 & 23,00 & 1,80 & 4,30 & 134,00 & 0,90 & 1,60 & 0,60 & 4,30 & 6,80 & 37,20 & 26,10 \\
\hline Cristal & 3 & 4,70 & 6,00 & 15,00 & 1,20 & 1,70 & 61,50 & 1,10 & 0,10 & 0,10 & 3,60 & 4,00 & 9,00 & 75,50 \\
\hline Cristal & 5 & 4,00 & 4,50 & 38,00 & 3,80 & 1,40 & 91,50 & 5,20 & 0,10 & 0,10 & 13,80 & 14,20 & 3,00 & 92,30 \\
\hline Cristal & 7 & 4,40 & 5,50 & 20,00 & 2,10 & 1,40 & 54,50 & 2,30 & 0,30 & 0,10 & 5,60 & 6,10 & 8,80 & 81,00 \\
\hline Piratini & 1 & 5,00 & 5,80 & 22,00 & 3,00 & 2,20 & 124,50 & 0,60 & 1,70 & 1,00 & 4,30 & 7,30 & 41,20 & 16,60 \\
\hline Piratini & 3 & 4,70 & 5,50 & 21,00 & 2,90 & 3,90 & 125,00 & 1,20 & 2,40 & 0,80 & 5,60 & 9,10 & 38,60 & 25,40 \\
\hline Piratini & 5 & 4,90 & 5,50 & 24,00 & 3,30 & 2,20 & 131,50 & 0,90 & 3,30 & 1,30 & 5,60 & 10,50 & 46,90 & 15,40 \\
\hline Piratini & 7 & 5,00 & 5,80 & 23,00 & 2,50 & 8,80 & 233,50 & 0,60 & 3,30 & 1,20 & 4,30 & 9,40 & 54,20 & 10,50 \\
\hline
\end{tabular}

$\mathrm{pH}=$ concentração de íons de $\mathrm{H}^{+}$; IND. SMP = índice SMP; M.O. = matéria orgânica; $\mathrm{P}$ = fósforo; $\mathrm{K}$ = potássio; $\mathrm{Al}=\mathrm{Alumínio;}$ $\mathrm{Ca}=$ Cálcio; $\mathrm{Mg}=$ Magnésio; $\mathrm{H}+\mathrm{Al}$ = hidrogênio + alumínio; $\mathrm{CTC}$ pH 7 = capacidade de troca de cátions; \% Sat. da CTC = porcentagem da saturação da CTC. 
(2.222 plantas por hectare), tendo sido adicionados $50 \mathrm{~g}$ de NPK (5-30-15) por planta.

Em cada povoamento, foi selecionada uma encosta de exposição norte, em que foram demarcadas três parcelas localizadas nos terços superior, médio e inferior da pendente. $\mathrm{O}$ tamanho das parcelas foi de $9 \mathrm{~m} \times 16 \mathrm{~m}$ para os povoamentos com um ano de idade e $9 \mathrm{~m} \times 14 \mathrm{~m}$ para as demais idades, equivalendo a quatro linhas de plantio e dez plantas em cada linha.

Todas as circunferências a 1,30 metro do solo (CAP) foram mensuradas utilizando-se fita métrica graduada e oito árvores em torno da árvore de CAP médio foram selecionadas para a mensuração de oito raios de suas copas. O raio da copa foi mensurado com trena, sendo considerado o raio a distância da projeção da copa no solo em relação ao fuste da árvore, sendo que, para cada árvore, foram tomadas oito medidas de raio. Assim, o DC foi estabelecido pelo dobro da média de oito raios mensurados.

Foram testados cinco modelos, descritos na Tabela 2, que comumente (Nutto et al., 2001; Tonini \& Arco Verde, 2005; Weber, 2007) são utilizados para estimar o diâmetro da copa (DC) em função do DAP. As equações foram avaliadas segundo o coeficiente de determinação ajustado $\left(\mathrm{R}^{2}{ }_{\mathrm{aj}}\right)$, o erro padrão da estimativa $\left(\mathrm{S}_{\mathrm{yx}}\right)$, o valor de $\mathrm{F}$ e a análise gráfica de resíduos. As equações logaritmizadas foram corrigidas pelo Índice de Meyer, sendo realizado também o recálculo do erro padrão da estimativa para unidades aritméticas. Tais estatísticas foram calculadas por meio das seguintes expressões:

$$
\mathrm{R}_{\mathrm{aj}}^{2}=1-\left(1-\mathrm{R}^{2}\right) \frac{\mathrm{n}-1}{\mathrm{n}-\mathrm{p}-1}
$$

Tabela 2. Modelos testados para a estimativa do DC em função do DAP.

Table 2. Models tested for the estimate of the DC in function of the DAP.

\begin{tabular}{cc} 
Modelo & Modelo \\
\hline $\mathrm{DC}=\beta_{0} \mathrm{DAP}^{\beta 1}$ & 1 \\
$\mathrm{DC}=\beta_{0}+\beta_{1} \mathrm{DAP}$ & 2 \\
$1 / \mathrm{DC}=\beta_{0+} \beta_{1}(1 / \mathrm{DAP})$ & 3 \\
$\mathrm{Ln}(\mathrm{DC})=\beta_{0}+\beta_{1} \ln (\mathrm{DAP})$ & 4 \\
$\mathrm{DC}=\beta_{0}+\beta_{1} \mathrm{DAP}+\beta_{2} \mathrm{DAP}$ & 5 \\
\hline $\mathrm{DC}=$ diâmetro de copa $(\mathrm{m}) ; \mathrm{Ln}=$ logaritmo neperiano; \\
$\begin{array}{l}\mathrm{DAP}=\text { diâmetro a } 1,30 \mathrm{~m} \text { do solo }(\mathrm{cm}) ; \text { bi }=\text { coeficiente do } \\
\text { modelo. }\end{array}$
\end{tabular}

$\mathrm{R}^{2}=\frac{\mathrm{SQR}}{\mathrm{SQT}}$

$\mathrm{S}_{\mathrm{yx} \%}=\frac{\sqrt{\frac{\sum\left(\mathrm{y}_{\mathrm{i}}-\hat{y}_{\mathrm{i}}\right)^{2}}{\mathrm{n}-\mathrm{p}}}}{y_{\mathrm{i}}}$ 100para o caso de equaçãolinear. (3

$\mathrm{S}_{\mathrm{yx} \%}=\frac{\sqrt{\frac{\sum\left[\mathrm{y}_{\mathrm{i}}-\left(\hat{\mathrm{y}}_{\mathrm{i}} \mathrm{IM}\right)\right]^{2}}{\mathrm{n}-\mathrm{p}}}}{\mathrm{y}_{\mathrm{i}}} 100$, para o caso de equação linearizada.(4)

$\mathrm{IM}=\mathrm{e}^{0,5 \mathrm{~S}_{\mathrm{yx}}^{2}}$

$\mathrm{F}=\frac{\mathrm{QMR}}{\mathrm{QMRe}}$

em que: $\mathrm{p}=$ número de coeficientes no modelo; $\mathrm{n}=$ número de observações; $\mathrm{SQR}=$ soma $\mathrm{de}$ quadrado de resíduos; SQT = soma de quadrado total; IM = Índice de Meyer; = base dos logaritmos neperianos; $\mathrm{QMR}$ = quadrado médio da regressão; $\mathrm{QMRe}=$ quadrado médio do resíduo; = quadrado do erro-padrão da estimativa em unidades logarítmicas, com o logaritmo neperiano para o caso; $y=$ diâmetro de copa, em $\mathrm{m}$.

Para a seleção da equação, foi utilizado o valor ponderado desses escores estatísticos, atribuindo-se pesos às estatísticas calculadas $\left(\mathrm{R}^{2}{ }_{\mathrm{aj}}, \mathrm{S}_{\mathrm{yx}}\right.$, valor de $\left.\mathrm{F}\right)$. As estatísticas foram ordenadas de acordo com sua eficiência, sendo atribuído peso 1 para a equação mais eficiente e pesos crescentes para as demais equações (ranking), conforme metodologia definida por Thiersch (1997).

Para verificar se as duas regiões de plantios (condições ambientais), a idade do povoamento e a sua interação possuíam a mesma relação de DC com o DAP, os dados foram submetidos à análise de covariância simples por Snedecor. Por observar que as diferenças foram significativas para ambos os fatores, a metodologia adotada foi o estudo individual para cada região do Estado. A análise de covariância simples, dada por um teste de F, foi calculada por meio da seguinte expressão:

$\mathrm{F}=\frac{\mathrm{QMD}}{\mathrm{QMT}}$

em que: QMT = quadrado médio do resíduo das regressões separadas e associadas a i graus de liberdade; $\mathrm{QMD}=$ quadrado médio da diferença, 
isto é, diferença entre a regressão individual e total para os grupos formados, e associado a i graus de liberdade.

Ao modelo escolhido para descrever o DC, foram adicionadas variáveis Dummy que, primeiramente, assumiram valores de 0 e 1 , conforme a idade do povoamento, de modo que: $\mathrm{Di}=1$, se a árvore estivesse presente na idade $\mathrm{i}$; e $\mathrm{Di}=0$, se a árvore estivesse ausente nessa idade i. Com esse método, foi possível expressar as regressões individuais ajustadas (em cada município) para as quatro idades avaliadas em função de uma regressão linear múltipla, representada pelas variáveis independentes na equação selecionada, de seguinte forma:

$\mathrm{DC}=\mathrm{f}(\mathrm{x} ; \mathrm{Di} ; \mathrm{Di} . \mathrm{x})$

em que: $\mathrm{DC}=$ diâmetro de copa $(\mathrm{m}) ; \mathrm{x}=\mathrm{DAP}$ (em sua forma natural ou transformada) $(\mathrm{cm})$; Di = Dummy (idade em estudo, onde $\mathrm{i}=1,3,5$ e 7); Di.x = interação variável Di com a variável $\mathrm{x}$.

Foram relacionados, também, os atributos químicos do solo, bem como os elementos meteorológicos. Os atributos químicos do solo avaliados foram: $\mathrm{pH}$, Índice SMP (Shoemaker, Mac lean e Pratt), teores de argila, matéria orgânica, quantidades de fósforo, potássio, alumínio, cálcio, magnésio, hidrogênio + alumínio, CTC a pH7 e a porcentagem da saturação da CTC.

Os elementos meteorológicos relacionados foram: precipitação anual acumulada ( $\mathrm{mm})$, temperatura média - máxima e mínima $\left({ }^{\circ} \mathrm{C}\right)$-, umidade relativa do ar (\%) e radiação solar global acumulada $\left(\mathrm{MJ} \mathrm{m}^{-2}\right)$. Os dados meteorológicos temperaturas máxima, mínima e média, umidade relativa do ar, precipitação e número de horas de insolação - foram adquiridos junto ao Instituto Nacional de Meteorologia (www.inmet.gov.br) da Estação Climatológica do município de Pelotas. A radiação global incidente foi estimada por meio da equação de Ängstrom modificada por Prescot (1940) e Penman (1948), conforme desenvolvimento matemático apresentado por Vianello \& Alves (2000) e com coeficientes ajustados para o município de Pelotas, por Steinmetz et al. (1999).

A relação entre os coeficientes (intercepto e angular) com os atributos químicos do solo e os elementos meteorológicos foi analisada pela técnica de seleção de variáveis Stepwise. Draper \& Smith (1980) citam que a regressão múltipla 'passo a passo' (Stepwise Multiple Regression) é um dos métodos mais recomendados para uma seleção criteriosa das variáveis explicativas.

\section{RESULTADOS E DISCUSSÃO}

A análise de covariância relevou a existência de diferenças significativas entre os fatores: condição ambiental, idade do povoamento e sua interação (Tabela 3). Isso se deve à diferença das relações entre o DC e o DAP ao longo dos anos e para os dois locais de plantios, bem como sua interação, produzindo, assim, um efeito dependente.

As estatísticas de avaliação dos modelos são apresentadas na Tabela 4. Para as diferentes idades e locais, os modelos que apresentaram o melhor desempenho não foram os mesmos, o que levou à ponderação das estatísticas de ajuste.

A ponderação dos escores estatísticos, juntamente com a análise gráfica de resíduos, conduziu à seleção do modelo número 4 para descrever a relação ao longo das idades dos povoamentos e para os locais de cultivo. Em relação aos resíduos, todas as equações resultantes tenderam a superestimar e subestimar os valores de diâmetro de copa para as árvores com menor e maior DAP, respectivamente.

As equações obtidas via o ajuste do modelo 4 apresentaram tendências nas estimativas (Figura 2); entretanto, os resíduos obtidos para os outros modelos apresentaram piores distribuições. Tal

Tabela 3. Análise de covariância (ANACOVA) do DC em função do DAP.

Table 3. Covariance analysis (ANACOVA) of DC in function of DAP.

\begin{tabular}{lccc}
\multicolumn{1}{c}{ Fonte variação } & GL & QM & F \\
\hline Modelo & 7 & 8,47361 & $29,43^{* *}$ \\
Condição ambiental (CA) & 1 & 4,46358 & $15,50^{* *}$ \\
\hline Idade do povoamento (IP) & 3 & 10,89803 & $37,85^{* *}$ \\
\hline CA $^{*}$ IP & 3 & 7,38586 & $25,65^{* *}$ \\
\hline Erro & 178 & 0,28793 & \\
Total & 185 & & \\
\hline
\end{tabular}

$\mathrm{GL}=$ graus de liberdade; $\mathrm{QM}=$ quadrado médio; $\mathrm{F}=$ valor de $\mathrm{F} ;{ }^{* *}=$ significativo a $1 \%$ de probabilidade, conforme a distribuição de Fisher. 
Tabela 4. Modelos ajustados e suas estatísticas de ajuste para estimativa do DC.

Table 4. Models adjusted and their adjustment statistics for estimate of DC.

\begin{tabular}{|c|c|c|c|c|c|}
\hline Município & Idade (anos) & Equação & $\mathbf{R}_{\mathrm{aj}}^{2} / \mathbf{I A}$ & $S_{y x}(\%)$ & $\mathbf{F}$ \\
\hline \multirow{20}{*}{ Cristal } & 1 & $\mathrm{DC}=1,5087^{\star} \mathrm{DAP}^{0,2699}$ & 0,18 & 10,84 & 1024,81 \\
\hline & 1 & $\mathrm{DC}=1,7747+0,1104^{*} \mathrm{DAP}$ & 0,18 & 11,04 & 6,15 \\
\hline & 1 & $\operatorname{Ln}(\mathrm{DC})=0,4313+0,2556^{\star} \operatorname{Ln}(\mathrm{DAP})$ & 0,21 & 11,11 & 4,99 \\
\hline & 1 & $\mathrm{DC}=2,1143+-0,0020^{*} \mathrm{DAP}+0,089^{*} \mathrm{DAP}^{2}$ & 0,11 & 11,02 & 3,00 \\
\hline & 1 & $1 / \mathrm{DC}=0,3217+0,5419^{*}(1 / \mathrm{DAP})$ & 0,11 & 11,03 & 3,74 \\
\hline & 3 & $\mathrm{DC}=0,6488^{\star} \mathrm{DAP}^{0,6068}$ & 0,53 & 13,66 & 628,70 \\
\hline & 3 & $\mathrm{DC}=0,9871+0,1620^{*} \mathrm{DAP}$ & 0,52 & 13,45 & 24,78 \\
\hline & 3 & $\operatorname{Ln}(\mathrm{DC})=-0,4631+0,6163^{\star} \operatorname{Ln}(\mathrm{DAP})$ & 0,55 & 14,03 & 26,68 \\
\hline & 3 & $\mathrm{DC}=0,3208+0,3024^{\star} \mathrm{DAP}-0,0070^{\star} \mathrm{DAP}^{2}$ & 0,48 & 13,96 & 38,67 \\
\hline & 3 & $1 / \mathrm{DC}=0,1703+2,1620^{*}(1 / \mathrm{DAP})$ & 0,49 & 13,84 & 26,20 \\
\hline & 5 & $\mathrm{DC}=0,3178^{\star} \mathrm{DAP}^{0,7856}$ & 0,39 & 19,66 & 302,99 \\
\hline & 5 & $\mathrm{DC}=0,3161+0,1582^{\star} \mathrm{DAP}$ & 0,62 & 19,46 & 35,34 \\
\hline & 5 & $\operatorname{Ln}(D C)=-1,4263+0,8892^{*} \operatorname{Ln}(\mathrm{DAP})$ & 0,64 & 20,41 & 68,61 \\
\hline & 5 & $\mathrm{DC}=-0,5659+0,3595^{\star} \mathrm{DAP}-0,0098^{\star} \mathrm{DAP}^{2}$ & 0,61 & 19,63 & 47,09 \\
\hline & 5 & $1 / \mathrm{DC}=0,1169+4,2352^{*}(1 / \mathrm{DAP})$ & 0,58 & 20,39 & 71,57 \\
\hline & 7 & $\mathrm{DC}=0,2485^{\star} \mathrm{DAP}^{0,9707}$ & 0,02 & 26,61 & 141,49 \\
\hline & 7 & $\mathrm{DC}=0,1771+0,2167^{*} \mathrm{DAP}$ & 0,58 & 13,88 & 54,28 \\
\hline & 7 & $\operatorname{Ln}(\mathrm{DC})=-1,0963+0,8506^{*} \operatorname{Ln}(\mathrm{DAP})$ & 0,72 & 13,91 & 41,76 \\
\hline & 7 & $\mathrm{DC}=2,8333-0,2311^{\star} \mathrm{DAP}+0,0179^{\star} \mathrm{DAP}^{2}$ & 0,69 & 13,85 & 80,80 \\
\hline & 7 & $1 / \mathrm{DC}=0,0862+3,3357^{*}(1 / \mathrm{DAP})$ & 0,63 & 15,01 & 30,32 \\
\hline \multirow{20}{*}{ Piratini } & 1 & $\mathrm{DC}=0,4604^{\star} \mathrm{DAP}^{0,5569}$ & 0,34 & 15,95 & 478,68 \\
\hline & 1 & $\mathrm{DC}=0,4242+0,1416^{\star} \mathrm{DAP}$ & 0,36 & 15,73 & 15,38 \\
\hline & 1 & $\operatorname{Ln}(D C)=-0,7329+0,5179^{*} \operatorname{Ln}(\mathrm{DAP})$ & 0,30 & 16,42 & 14,69 \\
\hline & 1 & $\mathrm{DC}=1,2636-0,3111^{\star} \mathrm{DAP}+0,0579 * \mathrm{DAP}^{2}$ & 0,39 & 16,03 & 13,97 \\
\hline & 1 & $1 / \mathrm{DC}=0,5035+2,0671^{*}(1 / \mathrm{DAP})$ & 0,41 & 15,06 & 9,90 \\
\hline & 3 & $\mathrm{DC}=0,7194^{\star} \mathrm{DAP}^{0,6477}$ & 0,45 & 13,13 & 647,75 \\
\hline & 3 & $\mathrm{DC}=0,9901+0,2206^{\star} \mathrm{DAP}$ & 0,46 & 13,05 & 22,45 \\
\hline & 3 & $\operatorname{Ln}(\mathrm{DC})=-0,3166+0,6377^{\star} \operatorname{Ln}(\mathrm{DAP})$ & 0,43 & 13,37 & 17,47 \\
\hline & 3 & $\mathrm{DC}=1,9201-0,0090^{\star} \mathrm{DAP}+0,0136^{\star} \mathrm{DAP}^{2}$ & 0,50 & 13,16 & 19,44 \\
\hline & 3 & $1 / \mathrm{DC}=0,1344+1,8544^{\star}(1 / \mathrm{DAP})$ & 0,44 & 13,25 & 11,07 \\
\hline & 5 & $\mathrm{DC}=0,5274^{\star} \mathrm{DAP}^{0,6404}$ & 0,56 & 14,05 & 624,12 \\
\hline & 5 & $\mathrm{DC}=0,8784+0,1411^{\star} \mathrm{DAP}$ & 0,56 & 14,08 & 32,48 \\
\hline & 5 & $\operatorname{Ln}(D C)=-0,6244+0,6299^{*} \operatorname{Ln}(\mathrm{DAP})$ & 0,54 & 14,37 & 23,95 \\
\hline & 5 & $\mathrm{DC}=0,5996+0,1921^{\star} \mathrm{DAP}-0,0022^{*} \mathrm{DAP}^{2}$ & 0,60 & 14,09 & 28,77 \\
\hline & 5 & $1 / \mathrm{DC}=0,1700+2,6876^{*}(1 / \mathrm{DAP})$ & 0,54 & 14,39 & 15,60 \\
\hline & 7 & $\mathrm{DC}=0,3831^{\star} \mathrm{DAP}^{0,7519}$ & 0,66 & 13,26 & 677,63 \\
\hline & 7 & $\mathrm{DC}=0,6248+0,1533^{\star} \mathrm{DAP}$ & 0,66 & 13,32 & 46,03 \\
\hline & 7 & $\operatorname{Ln}(\mathrm{DC})=-1,0513+0,7833^{*} \operatorname{Ln}(\mathrm{DAP})$ & 0,65 & 13,42 & 52,15 \\
\hline & 7 & $\mathrm{DC}=0,3719+0,1934^{\star} \mathrm{DAP}-0,0015^{\star} \mathrm{DAP}^{2}$ & 0,69 & 13,30 & 48,43 \\
\hline & 7 & $1 / \mathrm{DC}=0,0852+3,8999^{*}(1 / \mathrm{DAP})$ & 0,64 & 13,61 & 22,09 \\
\hline
\end{tabular}

$\mathrm{DC}=$ diâmetro de copa $(\mathrm{m}) ; \mathrm{Ln}=$ logaritmo neperiano; $\mathrm{DAP}=$ diâmetro a 1,30 metro do solo $(\mathrm{cm})$.

situação reforçou a escolha do modelo de número 4 para descrever a relação entre o DC e o DAP, cujo ajuste apresentou valores de $\mathrm{R}_{\text {aj }}^{2}$ entre 0,5 e 0,6 , e $\mathrm{S}_{\mathrm{yx}}$ de 13 a $28 \%$. Essas estatísticas de ajustes, razoavelmente satisfatórias, são em decorrência da grande variabilidade do DC em função do DAP, principalmente para as árvores com as maiores ou menores dimensões. Além disso, observa-se que o DC, além de sofrer influências internas nas árvores, ocasionadas pelo tamanho do DAP, sofre influência de fatores externos, tais como a competição entre árvores vizinhas e as condições ambientais. 


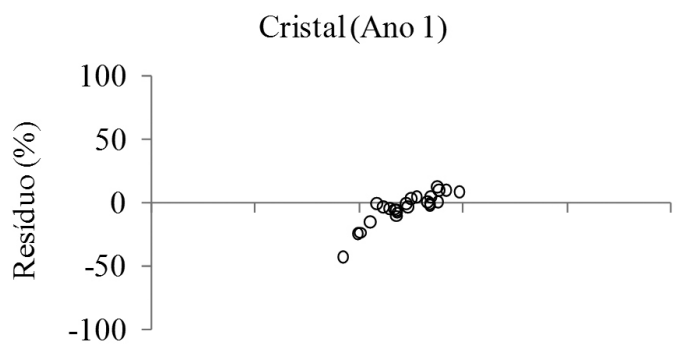

Cristal (Ano 3)

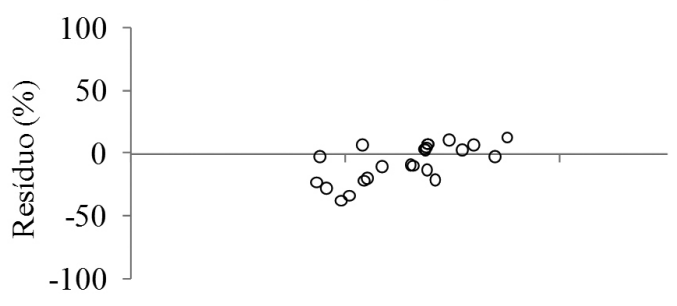

Cristal(Ano 5)

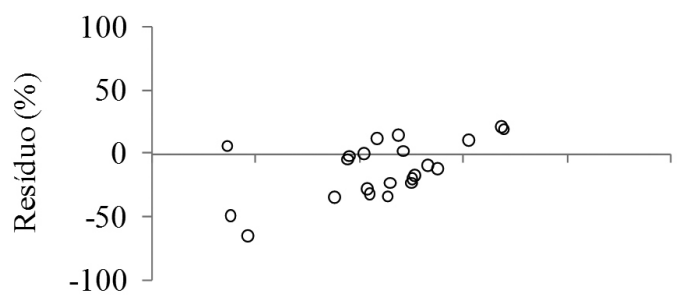

Cristal(Ano 7)

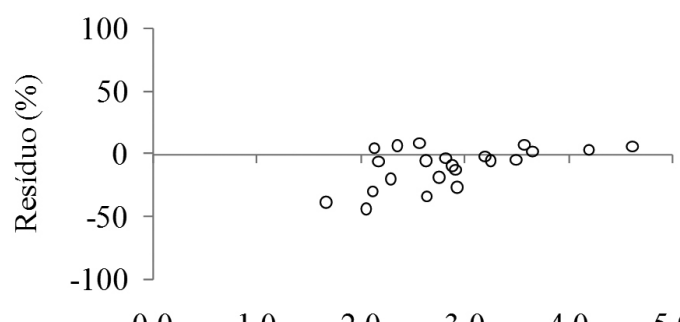

Diâmetro da copa $(\mathrm{m})$
Piratini(Ano 1)

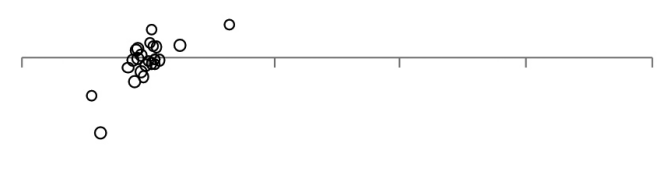

Piratini(Ano 3)

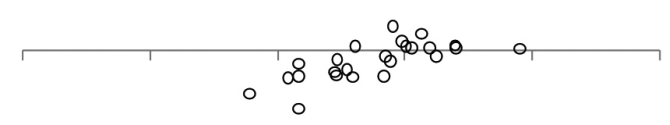

Piratini (Ano 5)

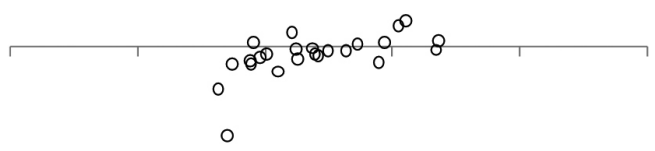

Piratini(Ano 7)

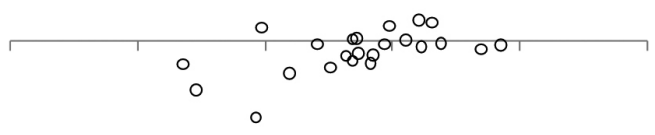

$1,0 \quad 2,0 \quad 3,0 \quad 4,0 \quad 5,0$

Diâmetro da copa (m)

Figura 2. Distribuição de resíduos do modelo ajustado $\operatorname{Ln}(\mathrm{DC})=\mathrm{b} 0+\mathrm{b} 1^{\star} \operatorname{Ln}(\mathrm{DAP})$. (Esquerda - município de Cristal; Direita - município de Piratini).

Figure 2. Distribution of residues of the fitted model $\operatorname{Ln}(D C)=b 0+b 1^{\star} \operatorname{Ln}(D A P)$. (Left - municipality of Cristal and right - municipality of Piratini).

Entretanto, os resultados obtidos estão de acordo com os observados por Orellana \& Koehler (2008), os quais obtiveram valores de $\mathrm{R}_{\text {aj }}^{2}$ de 0,58 para a relação do DC com o DAP para Ocotea odorífera (Vell.) Rohwere; também por Weber (2007), que observou valores de $\mathrm{S}_{\mathrm{yx}}$ entre 27,09 e 65,77 para a Mimosa scrabella Benth. Além disso, em termos absolutos, foi verificado que os ajustes da equação selecionada apresentaram erro inferior a $1 \mathrm{~m}$. Assim, o modelo selecionado proporcionou estimativas coerentes para todas as idades e para os dois locais de cultivo. 
Dawkins (1963) indica que a relação entre DC e DAP deve ser uma linha linear com coeficientes angulares positivos. Os resultados observados indicaram que o DC é maior à medida que aumenta o DAP, visto que todos os coeficientes angulares foram positivos; porém, para a acácia-negra, não há uma relação linear, mas logarítmica.

Além disso, foi observado, dentro de um mesmo ano, que o incremento em DC em função do DAP (IDC) diminuiu em diferentes intensidades, ou seja, reproduziu o efeito do modelo logarítmico, sendo maior o incremento de copa em relação aos menores DAP e menor para os maiores DAP (Figura 3). Ainda, a relação ao longo dos anos e entre os locais de cultivos (Cristal e Piratini) não foi a mesma, o que foi constatado pela análise de covariância simples e pela adição de variáveis Dummy no modelo selecionado para cada local de cultivo.

A análise de covariância com a adição de variáveis Dummy para o município de Cristal (Tabela 5) mostrou que, ao longo dos anos, os povoamentos com idade de 1,3 e 5 anos diferiram quanto ao intercepto em relação ao povoamento com 7 anos de idade; portanto, não estiveram no mesmo nível, $o$ que apenas refletiu na dimensão do tamanho da copa (Tabela 5; Figura 4A).

Em termos de relação entre o DC e o DAP, a análise de covariância revelou existirem diferenças entre o povoamento com 1 ano de idade e aquele com 7 anos, sendo a relação maior nos povoamentos de 3, 5 e 7 anos, quando comparados ao ano 1. Para o município de Piratini, somente foram identificadas diferenças significativas para o intercepto (Tabela 5), sendo que os povoamentos com 1 e 3 anos de idade diferiram quando comparados com os de 5 e 7 anos (Tabela 5; Figura 4B).

Diferenças significativas foram observadas para as regiões somente entre o intercepto, ou seja, os locais de cultivo (Serra do Sudeste e Encosta do Sudeste) influenciam no tamanho da copa. Assim, por meio da análise de covariância com adição de variáveis Dummy no modelo, para verificar o comportamento entre os locais de plantio dentro de uma mesma idade (Tabela 6), foi observado que os ambientes não diferiram no coeficiente angular, o qual expressa a relação entre o DC e o DAP, ou seja, apresentaram tendências de crescimento equivalentes.
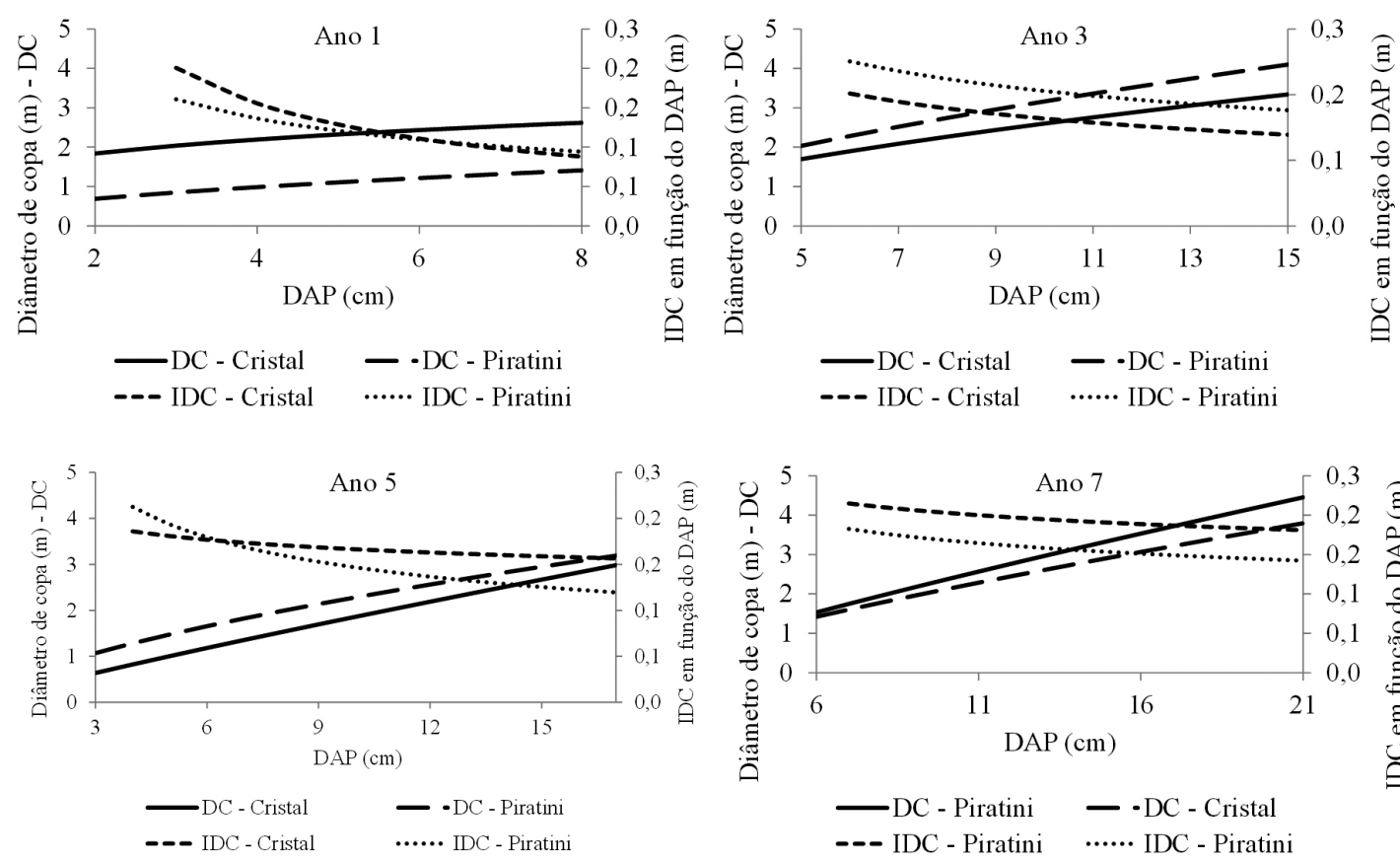

Figura 3. DC e IDC em função do DAP do modelo $\operatorname{Ln}(\mathrm{DC})=\mathrm{b} 0+\mathrm{b} 1^{\star} \operatorname{Ln}(\mathrm{DAP})$, para as idades e os municípios. Figure 3. DC and IDC in function of DAP of the model $\operatorname{Ln}(\mathrm{DC})=\mathrm{b} 0+\mathrm{b} 1^{\star} \operatorname{Ln}(\mathrm{DAP})$, for the ages and municipalities districts. 
Tabela 5. Análise de covariância do DC em função do DAP com a inclusão de variáveis Dummy. Table 5. Analysis of covariance of DC in function of DAP with the inclusion of variables Dummy.

\begin{tabular}{|c|c|c|c|}
\hline \multirow{2}{*}{ Fator de variação } & \multirow{2}{*}{ Graus de liberdade } & \multicolumn{2}{|c|}{ Quadrado médio } \\
\hline & & Cristal & Piratini \\
\hline Modelo & 7 & $0,7706^{* *}$ & $2,7973^{* *}$ \\
\hline Lnd & 1 & $2,1402^{* *}$ & $16,0446^{* *}$ \\
\hline D1 & 1 & $1,6491^{\star *}$ & $2,1446^{\star}$ \\
\hline D2 & 1 & $0,6044^{\star \star}$ & $1,3232^{\star}$ \\
\hline D3 & 1 & $0,6511^{\star *}$ & $0,0134^{\mathrm{ns}}$ \\
\hline D4 & 0 & - & - \\
\hline $\operatorname{Lnd}^{\star} \mathrm{D} 1$ & 1 & $0,2739^{\star *}$ & $0,0323^{\text {ns }}$ \\
\hline Lnd ${ }^{\star} \mathrm{D} 2$ & 1 & $0,0743^{\mathrm{ns}}$ & $0,0039^{\text {ns }}$ \\
\hline $\operatorname{Lnd}^{\star} \mathrm{D} 3$ & 1 & $0,0014^{\mathrm{ns}}$ & $0,0190^{\mathrm{ns}}$ \\
\hline Lnd $^{*}$ D4 & 1 & - & - \\
\hline Erro & 83 & 0,0237 & 0,0220 \\
\hline Total & 90 & 0,0818 & 21,4959 \\
\hline
\end{tabular}

$\mathrm{Ln}=$ logaritmo natural; $\mathrm{Di}=$ variável Dummy; ns = não significativo; ${ }^{*}=$ significativo a $5 \%$ de probabilidade; ${ }^{* *}=$ significativo a $1 \%$ de probabilidade; D1 $=1$ ano; $\mathrm{D} 2=3$ anos; $\mathrm{D} 3=5$ anos.
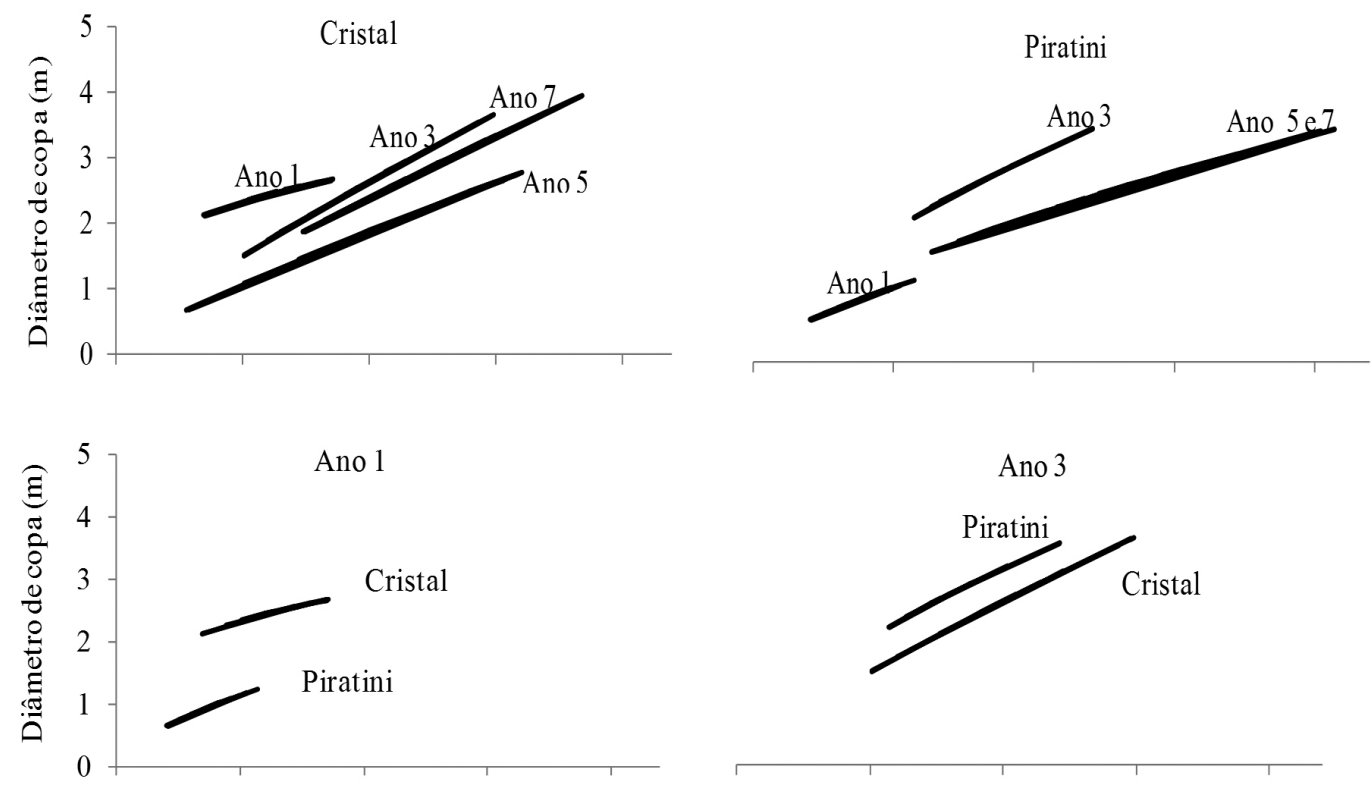

Ano 3
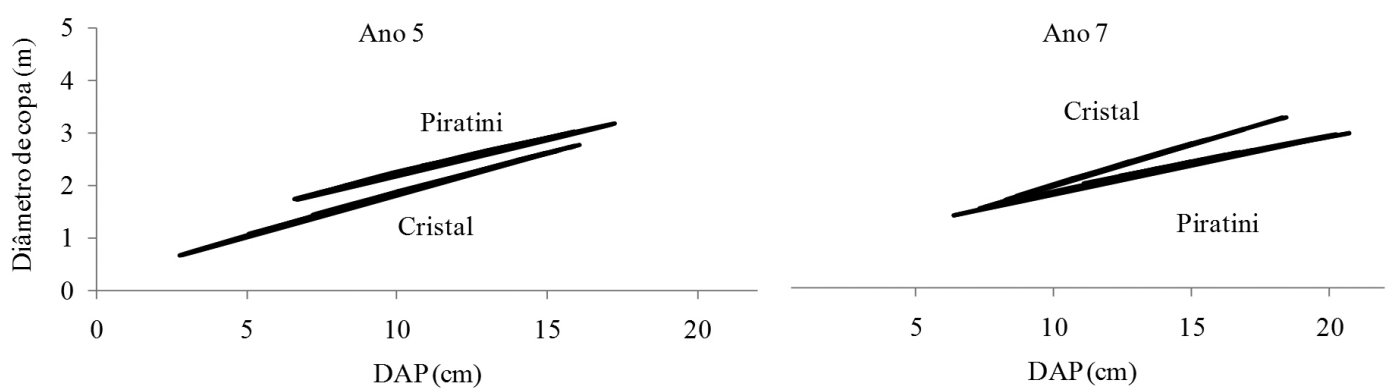

Figura 4. DC em função do DAP da acácia-negra originados pelo ajuste do modelo $\operatorname{Ln}(\mathrm{DC})=\mathrm{b} 0+\mathrm{b} 1^{\star} \operatorname{Ln}(\mathrm{DAP})$ para as idades nos municípios.

Figure 4. DC in function of DAP of the black wattle originated by the adjustment of the model $\operatorname{Ln}(D C)=b 0+b 1^{\star} \operatorname{Ln}(D A P)$ for the ages in the municipal districts. 
Tabela 6. Análise de covariância do DC em função do DAP da acácia-negra com a inclusão de variáveis Dummy. Table 6. Analysis of covariance of DC in function of DAP of the black wattle with the inclusion of variables Dummy.

\begin{tabular}{|c|c|c|c|c|c|}
\hline \multirow{2}{*}{$\begin{array}{l}\text { Fonte de } \\
\text { Variação }\end{array}$} & \multirow{2}{*}{$\begin{array}{c}\text { Grau de } \\
\text { liberdade }\end{array}$} & \multicolumn{4}{|c|}{ Quadrado Médio } \\
\hline & & Ano 1 & Ano 3 & Ano 5 & Ano 7 \\
\hline Modelo & 3 & $10,6093^{\star *}$ & $0,8960^{\star *}$ & $3,6772^{\star *}$ & $0,6463^{* *}$ \\
\hline Ln (DAP) & 1 & $7,2740^{* *}$ & $0,5928^{* *}$ & $3,2086^{* *}$ & $1,7961^{\star *}$ \\
\hline D1 & 1 & $3,2959^{* *}$ & $0,3030^{* *}$ & $0,3876^{\star *}$ & $0,1345^{*}$ \\
\hline D2 & 0 & --- & --- & --- & --- \\
\hline $\operatorname{Ln}\left(\mathrm{DAP}^{\star} \mathrm{D} 1\right)$ & 1 & $0,0394^{\mathrm{ns}}$ & $0,0002^{\mathrm{ns}}$ & $0,0810^{\mathrm{ns}}$ & $0,0085^{\text {ns }}$ \\
\hline $\operatorname{Ln}\left(\mathrm{DAP}^{\star} \mathrm{D} 2\right)$ & 0 & --- & --- & --- & --- \\
\hline Erro & 44 & 0,8591 & 0,0209 & 0,0314 & 0,0222 \\
\hline Total & 47 & 0,2440 & 0,0382 & 0,1063 & 0,0608 \\
\hline
\end{tabular}

${ }^{*}=$ significativo a $5 \%$ de probabilidade; ${ }^{* *}=$ significativo a $1 \%$ de probabilidade; ns = não significativo.

Entretanto, foram observadas diferenças no tamanho das copas, dadas pela diferença em todos os casos do intercepto (Figuras 4C, D, E e F). O tamanho das copas depende do local de plantio, ou seja, do sítio. Foi observado que os povoamentos com 1 e 7 anos de idade estabelecidos na Encosta do Sudeste apresentaram maiores tamanhos de copa, ao passo que os povoamentos com 3 e 5 anos de idade apresentaram os maiores tamanho de copa quando cultivados na Serra do Sudeste.

A diferença das relações do DC com o DAP, ao longo dos anos e entre as duas regiões, determinou a interação significativa da análise de covariância simples. Tal resultado pode ser uma função direta das condições do ambiente a que as plantas estão submetidas, tais como competição entre as plantas e efeitos dos atributos químicos e físicos do solo, e das condições meteorológicas ocorridas durante o ciclo de cultivo.

No sentido de buscar informações mais apropriadas para as diferentes respostas das relações entre o DC com o DAP em função do local de cultivo e da idade do povoamento, os coeficientes do modelo obtido foram relacionados com os atributos químicos do solo e com os elementos meteorológicos.

Para o coeficiente angular (CA), o método Stepwise resultou em três passos, incluindo, no primeiro, a variável mais correlacionada, a precipitação (Prec), e obtendo-se a equação: $\mathrm{CA}=0,35303+0,000055^{\star}$ Prec. $\mathrm{O}$ ajuste apresentou um coeficiente de determinação de $76,39 \%$ e um erro padrão da estimativa de $16,49 \%$ com todos os coeficientes da equação significativos. No segundo passo, foi inclusa a segunda variável que apresentou o maior coeficiente de correlação parcial, a temperatura do ar média máxima (Tmax), resultando em: $\mathrm{CA}=7,06673+0,000057^{\star}$ Prec $-0,28800^{\star}$ Tmax. $\mathrm{O}$ ajuste denotou um coeficiente de determinação de $91,83 \%$ e um erro padrão da estimativa de $10,63 \%$. No terceiro passo, a terceira variável a entrar no modelo foi a saturação da CTC, porém a mesma não apresentou coeficiente significativo $\left(a_{t}=0,0841\right)$, dado pelo teste de significância $t$ de Student, sendo, então, excluída do modelo.

Para o intercepto (Int), o método Stepwise resultou em dois passos, incluindo, no primeiro, a mesma variável que a verificada para o coeficiente angular. Assim, a seguinte equação foi obtida: Int $=0,06619-0,000135^{\star}$ Prec, resultando em um coeficiente de determinação de $77 \%$ e erro padrão da estimativa de 59,95\%. No segundo passo, a variável a entrar no modelo foi a temperatura média do ar, porém, a mesma não apresentou coeficiente de significativo $\left(a_{t}=0,1041\right)$, dado pelo teste de significância $t$ de Student, sendo, então, excluída do modelo.

Foi verificado que os sinais algébricos dos coeficientes da regressão foram positivos com a precipitação e negativos com a temperatura do ar média máxima para o coeficiente angular, e negativo com a precipitação para o intercepto, estando adequados à expectativa prévia, ou seja, relação direta com a água no solo e inversa com as temperaturas máximas do ar (Figura 5), visto que a temperatura do ar é um dos principais elementos meteorológicos que afetam o crescimento dos vegetais (Hodges, 

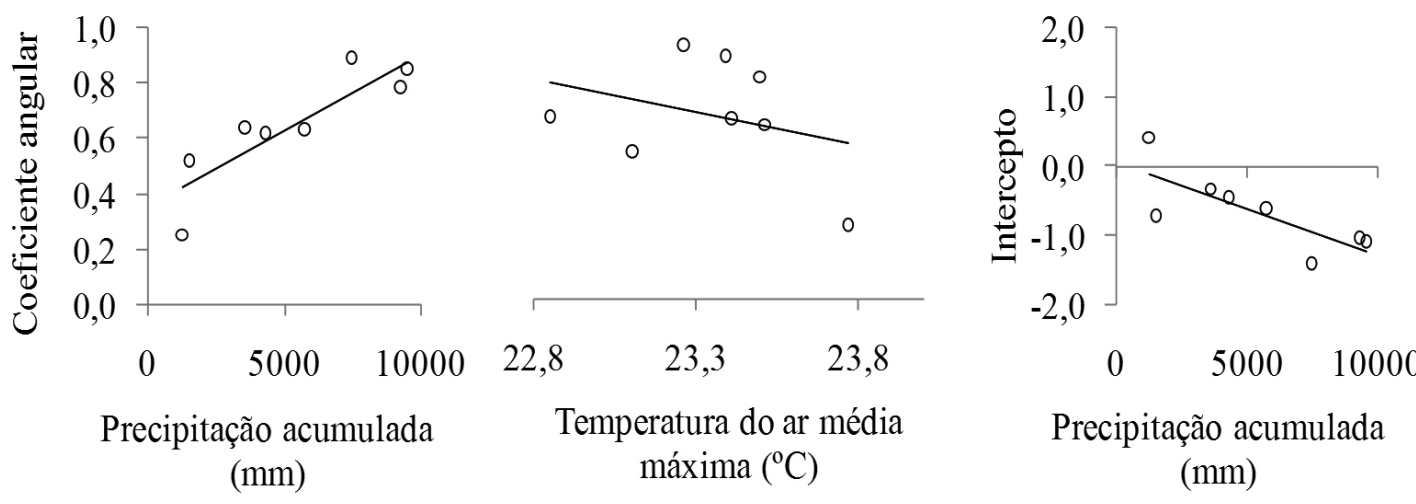

Figura 5. Relação do intercepto e coeficiente angular do modelo $\operatorname{Ln}(\mathrm{DC})=\mathrm{b} 0+\mathrm{b} 1^{\star} \operatorname{Ln}(\mathrm{DAP})$ com a precipitação acumulada e a temperatura do ar média máxima.

Figure 5. Relationship of intercept and angular coefficient the model: $\operatorname{Ln}(\mathrm{DC})=\mathrm{b} 0+\mathrm{b} 1^{\star} \operatorname{Ln}(\mathrm{DAP})$ with the accumulated precipitation and temperature of the air maximum average.

1991; Yan \& Hunt, 1999; Streck, 2002) e as reações bioquímicas da fotossíntese (Taiz \& Zeiger, 2004).

As temperaturas altas podem reduzir a assimilação de carbono da planta, em razão do aumento da transpiração e do fechamento dos estômatos. Por outro lado, em temperaturas baixas, tais como em dias com ocorrência de geadas, também ocorre redução da atividade fotossintética por certo período de tempo, em consequência do congelamento das células (Larcher, 2000). Isso corrobora com o que foi observado na relação entre o DC e o DAP.

A precipitação pluviométrica foi o fator principal que limita o crescimento de uma planta, visto que Sands \& Mulligan (1990), Blake \& Tschalplinski (1992) e Landsberg (2003) destacaram que a água é um dos fatores mais limitantes à produtividade florestal, por controlar a abertura e o fechamento estomático, e a absorção de nutrientes do solo, e por ser o meio no qual ocorrem as reações químicas e bioquímicas da fotossíntese. Do mesmo modo, também influencia na capacidade das árvores em atingir determinado tamanho de copa e na própria relação entre o DC e o DAP.

Pode-se destacar que, à medida que se acumula a precipitação pluviométrica, o tamanho das copas também tende a aumentar, bem como a relação entre o DC e o DAP. Ao passo que a temperatura média máxima do ar vem a influenciar de maneira negativa, ou seja, quanto maior a temperatura média do ar durante o ciclo de cultivo, menor a relação entre o DC e o DAP, e o tamanho das copas.

Assim, ficou evidenciado que, ao longo dos anos, os elementos meteorológicos - precipitação e temperatura média máxima - influenciam na relação entre o DC e o DAP, e também no tamanho das copas, explicando, dessa forma, as variações encontradas para as interações dos fatores de estudo. É válido lembrar que o impacto da influência desses elementos meteorológicos também dependerá do local, tendo em vista as inúmeras interações que ocorrem entre esses elementos com o ambiente, produzindo uma interação complexa entre o sistema solo-água-planta.

A fim de produzir um modelo único para a estimativa do DC em função do DAP, para cada local de plantio, foi gerado um modelo genérico, com a adição de variáveis Dummy, tendo em vista as diferenças entre os interceptos e os coeficientes angulares ao longo dos anos. Assim, para o município de Cristal, localizado na Encosta do Sudeste, foi obtida a equação:

$\operatorname{Ln}(\mathrm{DC})=-1,02529+0,82205^{\star} \operatorname{Ln}(\mathrm{DAP})+1,45665^{\star} \mathrm{D} 1+0,0993$ $1^{\star} \mathrm{D} 2-0,23867{ }^{\star} \mathrm{D} 3-0,56649^{\star} \operatorname{Ln}\left(\mathrm{DAP}{ }^{\star} \mathrm{D} 1\right)$

em que: $\mathrm{D} 1$ = povoamento com um ano de idade; D2 = povoamento com três anos de idade; D3 = povoamento com cinco anos de idade. 


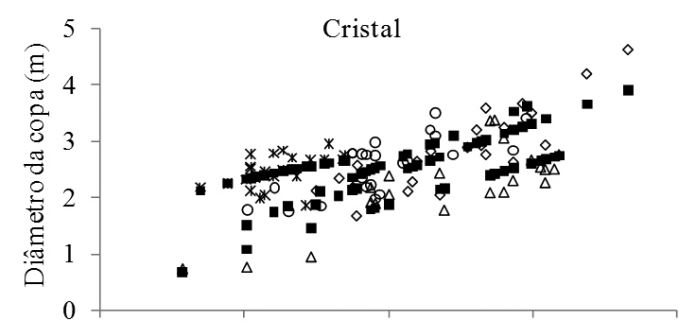

Cristal
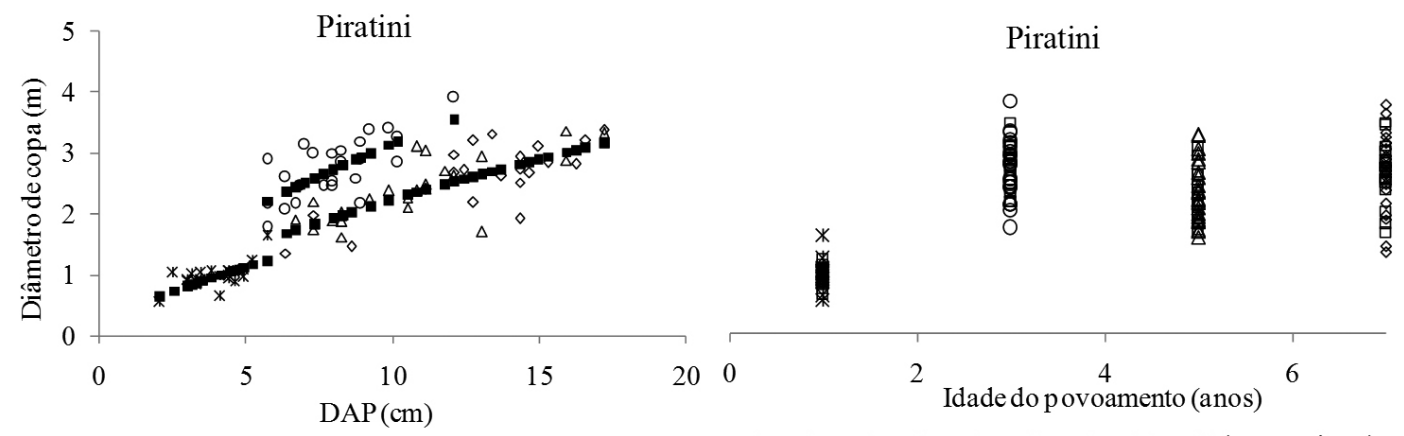

* Ano $1 \circ$ Ano $3 \triangle$ Ano $5 \diamond$ Ano 7 - Valores estimados $*$ Ano 1 OAno $3 \triangle$ Ano $5 \diamond$ Ano 7 Valores estimados
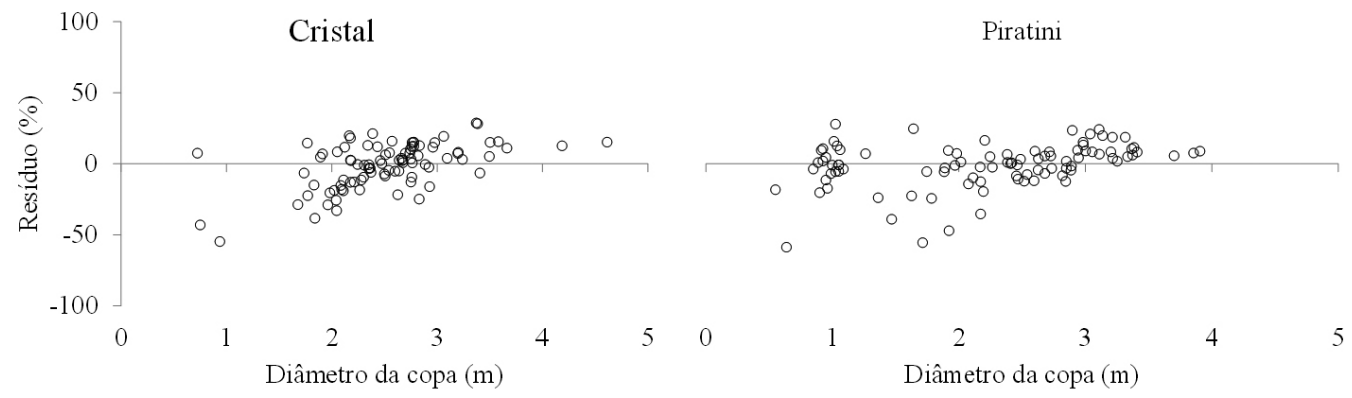

Figura 6. Desempenho e distribuição dos resíduos das equações genéricas ajustadas.

Figure 6. Acting and distribution of the residues of the adjusted generic equations.

A equação obtida resultou em um coeficiente de determinação de $72,28 \%$ e um erro padrão da estimativa de $17,41 \%$.

Para o município de Piratini, localizado na Serra do Sudeste, o modelo genérico obtido foi: $\operatorname{Ln}(\mathrm{DC})=-$ $0,65188+0,63505^{\star} \operatorname{Ln}(\mathrm{DAP})-0,24004^{\star} \mathrm{D} 1+0,34072^{\star} \mathrm{D} 2$

Em que: D1 = povoamento com um ano de idade; $\mathrm{D} 2$ = povoamento com três anos de idade; D3 = povoamento com cinco anos de idade.

O ajuste denotou um coeficiente de determinação de $90,77 \%$ e um erro padrão da estimativa de $21,34 \%$.

Além das boas estatísticas de ajustes, para os dois casos, as equações genéricas também apresentaram adequada distribuição de resíduos (Figura 6), ou seja, sem tendenciosidade; portanto, adequadas para o emprego em processos estimativos.

\section{CONCLUSÕES}

$\mathrm{O}$ modelo $\operatorname{Ln}(\mathrm{DC})=\mathrm{b} 0+\mathrm{b} 1^{\star} \operatorname{Ln}(\mathrm{DAP})$ é apropriado para o uso em estimativa do diâmetro da copa (DC) em função do diâmetro a 1,30 metro do solo (DAP).

O diâmetro da copa é maior à medida que aumenta o diâmetro a 1,30 metro do solo, de forma a atingir uma relação logarítmica.

O incremento em diâmetro de copa em função do diâmetro a 1,30 metro do solo diminui em diferentes intensidades, sendo maior o incremento de copa em relação aos menores diâmetros a 1,30 metro do solo e menor para os maiores diâmetros a 1,30 metro do solo. 
A relação do diâmetro de copa com o diâmetro a 1,30 metro do solo, ao longo da idade do povoamento e entre os locais de cultivos (Serra do Sudeste e Encosta do Sudeste), não é a mesma, produzindo efeitos dependentes para esses fatores de estudo.

Os coeficientes do modelo empregado para descrever o diâmetro da copa em função do diâmetro a 1,30 metro do solo são influenciados pela precipitação pluviométrica e pela temperatura média máxima do ar.

As equações genéricas geradas para descrever o diâmetro da copa em função do diâmetro a 1,30 metro do solo em cada condição ambiental são adequadas para a estimativa do diâmetro da copa ao longo das idades.

\section{AGRADECIMENTOS}

Os autores agradecem ao CNPq, pela bolsa de Pós-Graduação do autor Alexandre Behling, e à Empresa TANAC S.A., pela colaboração na pesquisa.

\section{STATUS DA SUBMISSÃO}

Recebido: 21 fev., 2013

Aceito: 31 out., 2013

Publicado: 30 jun., 2014

\section{AUTOR(ES) PARA CORRESPONDÊNCIA}

\section{Alexandre Behling}

Departamento de Ciências Florestais, Universidade Federal do Paraná - UFPR, CEP 80210-170, Curitiba, PR, Brasil e-mail: alexandre.behling@yahoo.com.br

\section{REFERÊNCIAS}

Blake TJ, Tschalplinski TJ. Water relations. In: Mitchell CP, Ford-Robertson JB, Hinckley T, Sennerby-Forsse $\mathrm{L}$, editor. Ecophysiolgy of short rotation forest crops. London: Elsevier Applied Science; 1992.

Costa E. A Influência de variáveis dendrométricas e morfométricas da copa no incremento periódico de Araucaria angustifólia (Bertol.) Kuntze, Lages, SC [dissertação]. Santa Maria: Universidade Federal de Santa Maria; 2011.
Dawkins HC. Crowndiameters: Their relationship to bole diameter in tropical trees. Commonweath Forest Review 1963; 42: 318-333.

Draper NR, Smith R. Applied regression analysis. New York: John Wiley \& Sons; 1966.

Fortes AB. Aspectos fisiográficos, demográficos e econômicos do Rio Grande do Sul. Porto Alegre: Livraria do Globo; 1956.

Hodges TF. Predicting crop phenology. Boca Raton: CRC; 1991.

Landsberg JJ. Physiology in forest models: History and the future. FBMIS; 2003.

Larcher W. Ecofisiologia vegetal. São Carlos: RIMA; 2000.

Leite LP, Zubizarreta-Gerendiain A, Robinson A. Modeling mensurational relationships of plantation - growth loblolly pine (Pinus taeda L.) in Urugay. Forest Ecology and Management 2012; 289: 455462. http://dx.doi.org/10.1016/j.foreco.2012.10.016

Miller EE, Norman JMA. Sunfleck theory for plant canopies. I. Lengths of sunlit segments a long a transect. Agronomy Journal 1971; 63: 735-738b. http://dx.doi. org/10.2134/agronj1971.00021962006300050024x

Mochiutti S. Produtividade e sustentabilidade de plantações de acácia-negra (Acacia mearnsii De Wild.) no Rio Grande do Sul. [Tese]. Curitiba: Setor de Ciências Agrárias, Universidade Federal do Paraná; 2007.

Monteith JL, Unsworth MH. Principles of environmental physics. London: Edward Arnold; 1990.

Nutto, L. Manejo do crescimento diamétrico de Araucaria angustifolia (Bert.) O. Ktze. baseado na árvore individual. Ciência floresta; 2001: 9-25.

Nutto L, Tonini H, Borsoi GA, Moscovich FA, Spathelf P. Utilização dos parâmetros da copa para avaliar o espaço vital em povoamentos de Pinus elliottii Engelm. Colombo: Embrapa; 2001.

Orellana E, Koehler AB. Relações morfométricas de Ocotea odorifera (Vell.) Rohwer Morphometric relations of Ocotea odorifera (Vell.) Rohwer. Revista Acadêmica Ciências Agrárias e Ambientais 2008; 6(2): 229-237.

Rio Grande do Sul. Macrozoneamento agroecológico e econômico do Estado do Rio Grande do Sul. Porto Alegre: Secretária da Agricultura e Abastecimento; 1994.

Sands R, Mulligan D. Water and nutrient dynamics and tree growth. Forest Ecology and Management 1990; 30: 91-111. http://dx.doi.org/10.1016/03781127(90)90129-Y

Sanquetta CR, Corte APD, Jacon AD. Crown area and trunk diameter relationship for tree species at a mixedaraucaria natural forest in the mid-southern Parana state, Brazil. Floresta 2011; 41(1): 63-72. 
Steinmetz S, Assis FN, Soares WR. Estimativa da radiação solar global a partir da insolação na região de Pelotas, Rio Grande do Sul. Agropecuária ClimaTemperado 1999; 2(1): 77-85.

Streck NA. A generalized nonlinear air temperature response function for node appearance rate in muskmelon (Cucumismelo L.). Revista Brasileira de Agrometeorologia 2002; 10(1): 105-111.

Penman HL, Natural evaporation from open water, bare soil and grass. Proceedings of the Royal Society of Landon 1948; 193: 120-145.

Prescott JA, Evaporation from a water surface in relation to solar radiation. Transactions of the Royal Society Science Australian 1940; 64: 114-118.

Taiz L, Zeiger E. Fisiologia vegetal. 3. ed. Porto Alegre: Artmed; 2004.

Thiersch A. Eficiência das distribuições diamétricas para prognose da produção de Eucalyptus camaldulensis [dissertação]. Lavras: Universidade Federal de Lavras; 1997.

Tonini H, Arco Verde MF. Morfologia da copa para avaliar o espaço vital de quatro espécies nativas da Amazónia, Brasília. Pesquisa Agropecuária Brasileira 2005; 40(47): 633-638. http://dx.doi.org/10.1590/ S0100-204X2005000700002

Vianello RL, Alves AR. Meteorologia básica e aplicações. Viçosa: UFV; 2000.

Weber KS. Manejo da bracatinga (Mimosa scrabella Benth.) baseado no crescimento diamétrico de árvores individuais. [dissertação]. Curitiba: Universidade Federal do Paraná; 2007.

Yan W, Hunt LA. An equation for modelling the temperature response of plants using only the cardinal temperatures. Annals of Botany 1999; 84(5): 607-614. http://dx.doi.org/10.1006/anbo.1999.0955 